\title{
Attention to Details Reduces Infection Rates in Patients with Continuous Flow Pumps
}

\author{
Nandini Nair ${ }^{1,2 *}$, Enrique Gongora ${ }^{3,4}$, Basar Sareyyupoglu3 ${ }^{3}$, Ian Collier ${ }^{1}$, Sherry Alvarado3, \\ Aasya Nasar ${ }^{5}$, Kenton Zehr ${ }^{3}$ \\ ${ }^{1}$ Division of Cardiology, Scott \& White Healthcare, Texas A \& M HSC College of Medicine, Temple, USA \\ ${ }^{2}$ Current Address: Department of Cardiology, Providence Sacred Heart Medical Center \& Children's Hospital, \\ Spokane, USA \\ ${ }^{3}$ Division of Cardiothoracic Surgery, Scott \& White Healthcare, Texas A \& M HSC College of Medicine, \\ Temple, USA \\ ${ }^{4}$ Current Address: Department of Cardiothoracic Surgery, Drexel University College of Medicine, \\ Philadelphia, USA \\ ${ }^{5}$ Department of Hospital Pharmacy, Scott and White Hospital, Texas A \& M HSC College of Medicine, \\ Temple, USA \\ Email: "nandini.nair@gmail.com
}

Received 1 January 2014; revised 1 February 2014; accepted 8 February 2014

Copyright (C) 2014 by authors and Scientific Research Publishing Inc.

This work is licensed under the Creative Commons Attribution International License (CC BY).

http://creativecommons.org/licenses/by/4.0/

\section{(c) (i) Open Access}

\section{Abstract}

The increased use of mechanical circulatory support has led to a rise in infectious complications in the recent years predisposing this population of patients to higher morbidity and mortality. In this study, we report a significantly lower rate of driveline infections of 0.12 episodes/patient-year as compared to the rates reported in the existing literature in patients with the HMII (Heartmate II). The study is limited by the fact that it is based on a small population of patients and was conducted retrospectively making recall bias hard to rule out. It is strictly restricted to one type of continuous flow pump (HMII) solely to decrease variations in the data reviewed. The exact cause of the low infection rate noted in this study is difficult to define. However, detailed teaching to the caregivers and the patient as well as close follow-ups in the perioperative period may substantially contribute to the outcome noted. Continued research limited to similar continuous flow ventricular assist devices in larger study populations would shed light on defining causes of infections in this population and developing robust algorithms to prevent such complications.

\section{Keywords}

Driveline Infections, Continuous Flow Pumps

\footnotetext{
${ }^{*}$ Corresponding author.
}

How to cite this paper: Nair, N., Gongora, E., Sareyyupoglu, B., Collier, I., Alvarado, S., Nasar, A. and Zehr, K. (2014) Attention to Details Reduces Infection Rates in Patients with Continuous Flow Pumps. Open Journal of Organ Transplant Surgery, 4, 15-22. http://dx.doi.org/10.4236/ojots. 2014.42003 


\section{Introduction}

The humble beginnings of mechanical circulatory support systems in non-human systems since the 1930s led to the first ever pneumatic pump in a patient that prolonged life in the 1960s [1]. Further research and development in this area resulted in the first Novacor pump being placed in 1984 as a bridge to transplant [2]. Mechanical circulatory devices have since evolved as mainstream therapy over the last few decades with accelerated growth in the recent past [3]. The use of ventricular assist devices has consequently evolved from being temporary supports to life-long use as destination therapy. Increasing use of ventricular assist devices (VADs), in the wake of donor organ shortage and higher incidence of end stage heart failure, has led us to the era of infectious complications post device placement. Infections of the driveline increase mortality and morbidity, increase number of hospital visits and length of hospital support as well as the possible need for surgical interventions. Post implantation, driveline infections increase healthcare costs, lower quality of life, and lead to poor outcomes in the long run making VADs seem like a less attractive option.

VAD infections have been defined, as per the ISHLT consensus document [4], as being of 3 types: (a) VAD-specific infections, (b) VAD-related infections, and (c) non-VAD infections. Hannan et al. [4] have put forth definitions to delineate etiology of the different VAD infections as well as their impact on outcomes. VAD-specific infections are those specific to patients with VADs, involving device hardware such as pump/ cannula/pocket and percutaneous driveline. VAD-related infections are those that are not limited to VAD patients, but include mediastinitis and infectious endocarditis that bear a definite relationship in time and space to presence of VADS; non-VAD infections are typically all infections in the VAD patient population studied. Therefore, using these definitions VAD infections may be better classified and the incidence of VAD specific infections possibly be accurately reported. The incidence reported varies widely because of differences in the types of VADS studied and the definitions used to qualify infection [5]-[14]. The most recent retrospective study by Nienaber et al. [15] using the consensus document definitions [4] showed that in a population of 247 patients with average support of 1.5+/- 1.0 years (94\% had Heartmate II (Thoratec Corporation, Pleasanton, CA, USA) with $62 \%$ being on destination therapy) the infection rate was $47 \%$ (driveline); $24 \%$ (VAD related bloodstream); and 22\% (non VAD related bloodstream). Most common pathogens were staphylococci followed by nosocomial gram negative bacilli.

In our study, we report a remarkably low incidence of VAD specific infections as compared to the existing literature. The study population consisted of $100 \%$ Heartmate II patients with approximately 55\% as destination therapy. Retrospective chart review was done from June 2010 to June 2013.

\section{Materials and Methods}

This project was approved by the Scott \&White Memorial Hospital institutional review board (project id number 130206).

\subsection{Materials}

- Surgical hats, masks, and sterile outerwear as appropriate for all individuals in the room, including the patient.

- 1 Poly-Reinforced Sirus ${ }^{\circledR}$ (Mediline Mudeliein, Il, USA) Surgical Gown, Large, X-Long.

- 3 Curity $^{\mathrm{TM}}$ (Covidien, Dublin, Ireland) Gauze Sponges, 12 Ply, $4 \times 4$ in.

- 1 Tegaderm ${ }^{\text {TM }}$ (3 m, St Paul, Minnesota, USA) Sterile Dressing Film, $6 \times 8$ in.

- 1 Tegaderm ${ }^{\mathrm{TM}}$ (3 m, St Paul, Minnesota, USA) Sterile Dressing Film, $4 \times 4.75$ in.

- 2 Excilon $^{\mathrm{TM}} \mathrm{AMD}^{\mathrm{TM}}$ (Covidien, Dublin, Ireland) Antimicrobial Drain Sponges.

- 2 Pairs of Sterile Gloves.

- 1 Container 0.9 Sodium Chloride Irrigation, USP, $250 \mathrm{~mL}$.

- 1 Container Scrub-Stat ${ }^{\mathrm{TM}}$ (Ecolab, Mississuaga Ont, Canada) 2\% Chlorhexidine Gluconate, $118 \mathrm{~mL}$.

\subsection{Methods}

\subsubsection{Surgical Construction of Driveline Pocket}

An adequately sized pocket was created under the rectus abdominis sheath to avoid leaving any dead space around the pump body and driveline. During surgical implantation, care was taken to leave the body of the pump and driveline covered with an antibiotic soaked surgical sponge until the time of implantation. A tunneler was 
used in a curved fashion to travel as much distance as possible from the rectus sheath in the right upper abdominal quadrant until the driveline was penetrating inside out. Only the silastic part of the driveline was exposed outside the skin leaving the velour part completely inside the body. The silastic part of the driveline has a smoother surface compared to velour part providing a better skin attachment leading to less dehiscence and presence of fewer bacteria. Polypropylene sutures were placed at the driveline entry site to anchor skin opening and one of the polypropylene sutures was tied around the driveline to secure the driveline and decrease its movement during the immediate postoperative period. The secure suture was left in place for a month postoperatively and removed in the outpatient clinic once the driveline was found to safely adhere to the skin.

\subsubsection{Dressing Change Protocol}

Our dressing change protocol does not vary from the clinic to the home setting, and dressing changes are always administered by a trained caregiver. All caregivers are trained post implantation by the VAD coordinators.

Prior to initiating the dressing change a sterile environment is established which entails that all individuals (including the patient) within the immediate area of the dressing change wear surgical masks and hats.

Once sterility is established, the equipment used for the dressing change is opened and three containers with Curity ${ }^{\mathrm{TM}}$ Gauze Sponges are assembled. In the first container of gauze sponges (container 1), $125 \mathrm{~mL}$ of $0.9 \%$ sodium chloride irrigation and $10 \mathrm{~mL}$ of Scrub-Stat ${ }^{\mathrm{TM}} 2 \%$ antimicrobial soap is placed. In the second container (container 2), $125 \mathrm{~mL}$ of $0.9 \%$ sodium chloride irrigation fluid is added. The third container (container 3 ) is filled with dry sponges. Container 1 functions as the wash, container 2 functions as the rinse, and container 3 serves as the dry sponge reserve.

After the cleaning agents are made ready for use, the used dressing is removed from the patient. The first of the double pair of gloves is discarded following the disposal of the used dressing. The exit site is further cleaned and washed using gauze sponges from container one. Before use, each gauze sponge is squeezed in order to remove all excess cleaning solution. Then, the driveline exit site is wiped using circular motions in a counterclockwise fashion taking care not to use a gauze sponge more than once. After each wiping motion, the next wipe is begun with a new gauze sponge starting with the tissue closest to the driveline exit site, and wiping outward with each circular wiping motion until the entire area of the driveline has been cleaned. After allowing a few minutes for the soap to set, this exact step is repeated with gauze sponges from container 2.

When the entire driveline site has been rinsed with gauze sponges from container 2, gauze sponges from container 3 are used to dry the site. During the drying stage of our protocol driveline exit site is dried in the same inside-outward circular fashion until the entire area is dry.

When drying is complete, one antimicrobial drain sponge is placed around the driveline cord. The driveline cord around the first drain sponge is looped, because this lessens the chance of pressure on the driveline followed by a second antimicrobial sponge around the driveline cord on top of the loop in the opposite orientation to the first. A large Tegaderm ${ }^{\mathrm{TM}}$ film $(8 \times 6$ in) is used to cover the driveline area in order to secure the driveline. Any area not completely covered by the large film, is covered by a smaller extra Tegaderm ${ }^{\mathrm{TM}}$ film $(4 \times 4.75 \mathrm{in})$. After the driveline has been completely covered an $\mathrm{d}$ secured, all gloves and sterile equipment may be removed. An abdominal binder is used by all patients to immobilize the driveline.

Follow up of patients was either until death or explantation of device for transplantation, or until the study period terminated.

\section{Results}

\subsection{Study Population and Retrospective Analyses}

The baseline characteristics of the study population are shown in Table 1. Thirty-six patients (32 males and 4 females) were included in the retrospective analyses: $39 \%$ of the subjects had ischemic cardiomyopathy, $39 \%$ had diabetes, $55 \%$ were on destination therapy, $6 \%$ had peripheral vascular disease, and $45 \%$ were smokers at the time of implantation. The average age was 56 years at implant and average length of pump support was 207 days; $8 \%$ of the population underwent pump exchange. Ethnicity of patients were predominantly Caucasian (78\%) followed by $11 \%$ African American, $8 \%$ Hispanic and 3\% Asian.

\subsection{Period of Retrospective Analyses}

In the 36 patients who were retrospectively studied, drive line infections were noted only in 3 patients thus far. 
Table 1. Baseline characteristics of study subjects.

\begin{tabular}{lc}
\hline \multicolumn{1}{c}{ Characteristic } & Percentage expressed in parentheses \\
\hline Male & $\mathbf{3 2}(\mathbf{8 9})$ \\
Female & $4(11)$ \\
Age at Implantation & $56 \pm 15$ \\
Ischemic & $14(39)$ \\
Non-Ischemic & $22(61)$ \\
Diabetes Mellitus (DM) & $14(39)$ \\
Hypertension & $21(58)$ \\
Peripheral Vascular Disease (PVD) & $2(6)$ \\
Pump Exchange & $3(8)$ \\
Length of Pump Support (LOPS) & $207 \pm 191$ \\
Bridge-to-Transplant (BTT) & $16(44)$ \\
Destination Therapy (DT) & $20(56)$ \\
History of Tobacco Use & $16(44)$ \\
\hline
\end{tabular}

No driveline infection related deaths occurred in the period reviewed. In the period of retrospective analyses 8 non-infection related deaths were noted. One patient had faced imminent amputation and hence pump support was withdrawn by family at 2 weeks status post implant. Two patients died of intracranial bleeds at 5 and 10 months of support. Three patients died of complications from right ventricular failure at 2, 7 and 8 months post implantation. Two patients died of intraoperative complications 1 day and 4 weeks post implantation. In the population that survived as bridge to transplant no infection has been noted. All three patients who developed infections were on destination therapy. Characteristics of patients who had infection are summarized in Table 2. Patient 1 had a driveline infection at 435 days of support with no blood stream involvement and was treated with Ciprofloxacin 400 iv q12 for 2 weeks. She was then transitioned to po ciprofloxacin $\times 4$ weeks. The patient had ongoing pain at 1 year post implantation hence a minor surgical procedure was performed to relieve pain by releasing a nerve entrapment. It is unclear if this led to a infective process that affected the driveline as this event could have interrupted the healing process.

Patient 2 had a driveline infection after an ileostomy was performed for an ischemic bowel injury. The bowel injury was caused by discontinuation of power supply to the pump inadvertently by the patient for a few minutes while switching from battery pack to power outlet. This event may have precipitated the driveline infection. The treatment was Vancomycin IV $\times 2$ weeks, Flagylpo $\times 2$ weeks, Daptomycin IV $\times 4$ weeks. This patient has had oral antibiotic suppression therapy with doxycycline and amoxicillin/clavulanate ever since the infection on an ongoing basis. Patient 2 also needed surgical debridement and placement of a wound vac as part of the treatment to enhance wound healing and growth of granulation tissue.

Patient 3 had a driveline infection $>1$ year post implantation secondary to non-compliance with adequate dressing changes. Intravenous antibiotic treatment was started after which patient had to be transferred to another transplant center due to insurance issues. Table 3 shows details of antibiotics and sensitivities of the microorganisms. Patient 2 required surgical debridement once. Driveline infections noted in the 3 patients in this study were all classified as superficial and involved no abscess formation. All patients suspected of having infections underwent additional radiographic, ultrasound and computed tomographic imaging to exclude abscess formation. Transthoracic and/or transesophageal echocardiography was performed if there was any clinical suspicion of endovascular seeding. All pump exchanges reported in this study population were performed due to thrombus formation.

\subsection{Incidence of Driveline Infection}

The incidence of driveline infections in this study is $8.3 \%$ and it translates to 0.12 episodes per patient year. 
Table 2. Characteristics of the patients with driveline infections.

\begin{tabular}{|c|c|c|c|}
\hline & Patient 1 & Patient 2 & Patient 3 \\
\hline Gender & Female & Female & Male \\
\hline Age (y) & 49 & 25 & 59 \\
\hline Ischemic & yes & yes & yes \\
\hline Non-Ischemic & No & No & No \\
\hline DM & Yes & Yes & Yes \\
\hline HTN & Yes & Yes & Yes \\
\hline PVD & Yes & No & Yes \\
\hline Exchange & No & No & No \\
\hline LOPS & 435 & 699 & 420 \\
\hline BTT/DT & DT & DT & DT \\
\hline Tobacco Use & No & No & No \\
\hline BMI & 35 & 40 & 29 \\
\hline
\end{tabular}

LOPS: length of pump support.

Table 3. Driveline infections-susceptibility and treatment.

\begin{tabular}{ccccc} 
& Time of onset of infection & Organism & Duration of Antibiotic Treatment & Sensitivity \\
\hline Patient 1 & $90-180$ days & $\begin{array}{c}\text { Methicillin Resistant } \\
\text { Staphylococcus aureus }\end{array}$ & Vancomycin IV $\times 2$ weeks & $\begin{array}{c}\text { Doxycyline/Clindamycin/ } \\
\text { Bactrim/Vancomycin }\end{array}$ \\
& & Klebsiellapneumoniae $\times 2$ weeks & Daptomycin iv $\times 4$ weeks \\
Patient 2 & $365-880$ days & Pseudomonas aeroginosa & Ciprofloxacin iv $\times 2$ weeks & Resistant to ampicillin \\
Patient $3{ }^{*}$ & $365-880$ days & Pseudomonas aeroginosa & Cefepime IV bid $\times 6$ weeks & Pan susceptible \\
\hline
\end{tabular}

*Patient was transferred to another transplant center.

Figure 1 is a graphic representation of the incidence over time. This is a remarkably low rate of driveline infections as compared to that reported in the literature from studies on HMII patients (1.65 episodes per patient year) [16].

\section{Discussion}

Infections in LVAD recipients have been studied in a variety of settings using different devices, as well as different classifications and definitions of infections and variable study populations, mostly in a retrospective fashion. This has led to considerable variation in the incidence of VAD specific infections reported. In this study we have addressed the incidence of driveline infections in a small population of patients with a single type of continuous flow left ventricular assist device (HMII). Our study is limited by the fact that it is based on a small population of patients and was conducted retrospectively. Hence, information recall bias cannot be ruled out. Our patient population was severely ill with $>75 \%$ of the study subjects being in INTERMACS $1-2$ at the time of implantation. Our study shows a markedly low incidence of driveline infections ( 0.12 episodes per patient year) the exact cause of which is difficult to pinpoint. Bomholt et al. in their study [16], which also included only HMII recipients, show an incidence of 1.65 episodes per patient per year. Consistent with our findings they also 


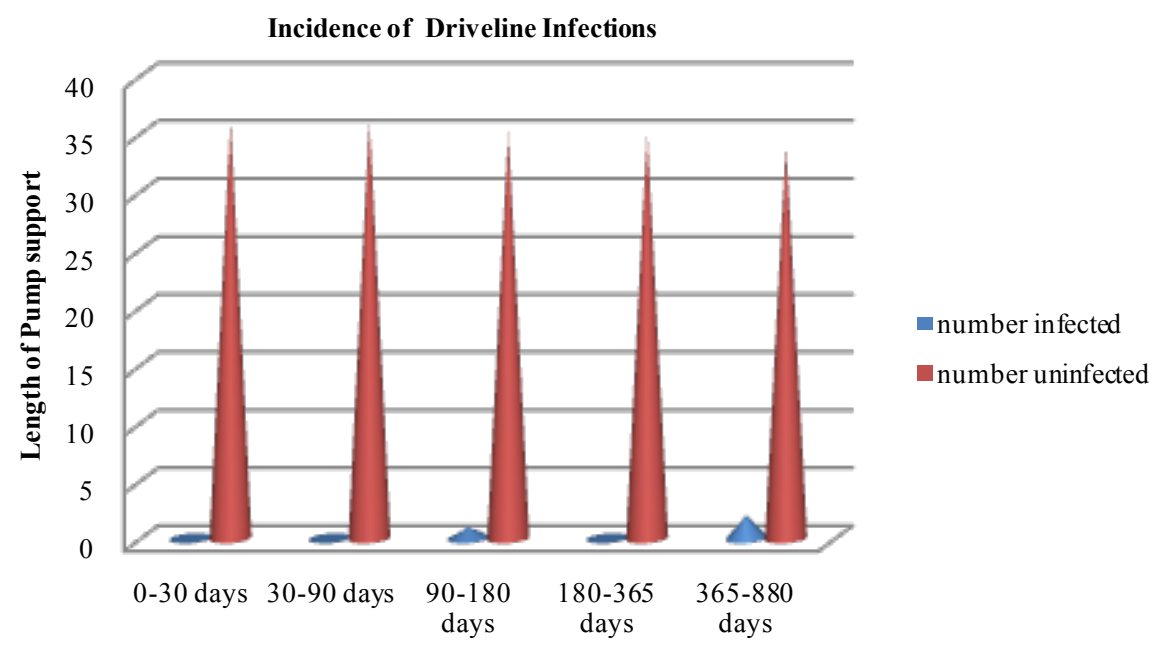

Figure 1. Incidence of driveline infections through the study period.

report only superficial infections in this population. The etiology noted was also secondary to infection arising from skin or gastrointestinal pathogens, which is in agreement with our findings. In another study by Loforte et al. [17], in a small population of 18 HMII recipients, two patients were reported to have a drive line infection which amounts to $16.7 \%$ in a follow up period of $217+/-212.3$ days versus our study which shows a driveline infection rate of 3/36 (8.4\%) for a pump support duration of $207 \pm 191$ days (Figure 1). In a bridge to transplant study [18], which focused on 102 HMII recipients, a $24.5 \%$ driveline infection was noted. A recent multicenter study [19] showed in a prospective follow up of up to 400 days status post implantation no differences in incidence of driveline infections in pulsatile versus continuous flow pumps. Abicht et al. [20], however, report an advantage by exchanging HMII with Heart ware LVAD because of the reduced size and flexible driveline of the latter in patients with complex driveline or pump infections. Gordon et al. [19] show a biphasic spurt of VAD infections such as $<60$ days post surgery and another phase around 210 - 240 days that constituted much fewer cases. The effect of depression on post implantation infection was an interesting aspect that was highlighted in the study by Gordon et al. [19].

Therefore, our study shows a lower rate of VAD infections ( 0.12 episodes per patient year) as compared to the numbers documented in literature (1.65 episodes per patient year) [16]. The study has its limitations as discussed above. In addition, the limited size makes rigorous statistical analyses of risk factors impractical. Review of literature and the experience in our center may highlight the importance of compliance with frequency and technique of dressing change, education of the caregiver and the patient and the use of regular antibiotic prophylaxis. This study has strictly focused on one type of continuous flow pump namely the Heartmate II in order to reduce variations in the data reviewed. It is also possible that meticulous construction of the driveline pocket and the placement of the polypropylene suture to minimize motion contribute to reduced driveline infection. Additionally our protocol calls for use of an abdominal binder to secure the driveline in place. Further research limited to similar continuous flow ventricular assist devices in larger study populations would shed light on developing robust algorithms. The most recently published algorithm by Nienaber et al. [15] would be a good start to assess risk factors and plan management of VAD infections to help improve outcomes as we plunge into the era of mechanical circulatory support systems. We propose a system of rigorous education for the caregiver and the patient on driveline dressing change, antibiotic prophylaxis in the perioperative period and close follow up in the early postoperative period up to 90 days.

\section{Author Contributions}

Nandini Nair, MD, Ph.D. — Concept/design/Data analysis/interpretation and Drafting article.

Enrique Gongora, MD—Concept/design/Data analysis/Interpretation.

Basar Sareyyupoglu, MD—Data analysis/Interpretation.

Ian Collier-Data collection and Drafting article.

Sherry Alvarado, MS, MBA—Data collection/analysis. 
Aasya Nazar, D. Pharm, MSc—Data Collection.

Kenton Zehr, MD—Concept/Data analysis/Critical revision of article.

\section{Funding}

This study was supported by an internal grant to Dr. Nandini Nair from the Division of Cardiology, Scott and White Memorial Hospital, Temple, Texas. Ian Collier was supported by a summer program (NSF-STEP) from the National Science Foundation.

\section{References}

[1] DeBakey, M.E. (1971) Left Ventricular Bypass Pump for Cardiac Assistance. American Journal of Cardiololgy, 27, 311. http://dx.doi.org/10.1016/0002-9149(71)90076-2

[2] Portner, P., Oyer, P. and McGregor, C. (1985) First Human Use of an Electrically Powered Implantable Ventricular Assist System. Artificial Organs, 9, 36.

[3] Kirklinand, J.K. and Naftel, D.C. (2008) Mechanical Circulatory Support: Registering a Therapy in Evolution. Circulation: Heart Failure, 1, 200-205. http://dx.doi.org/10.1161/CIRCHEARTFAILURE.108.782599

[4] Hannan, M.M., Husain, S., Mattner, F., et al., (2011) Working Formulation for the Standardization of Definitions of Infections in Patients Using Ventricular Assist Devices. The Journal of Heart and Lung Transplantion, 30, 375-384. http://dx.doi.org/10.1016/j.healun.2011.01.717

[5] Gordon, R.J., Quagliarello, B. and Lowy, F.D. (2006) Ventricular Assist Device-Related Infections. The Lancet Infectious Disease, 6, 426-437. http://dx.doi.org/10.1016/S1473-3099(06)70522-9

[6] Baddour, L.K., Bettmann, M.A., Bolger, A.F., et al. (2003) Nonvalvular Cardiovascular Device-Related Infections. Circulation, 108, 2015-2031. http://dx.doi.org/10.1161/01.CIR.0000093201.57771.47

[7] Gordon, R.J., Weinberg, A.D., Pagani, F.D., et al. (2013) Prospective, Multicenter Study of Ventricular Assist Device Infections. Circulation, 127, 691-702. http://dx.doi.org/10.1161/CIRCULATIONAHA.112.128132

[8] Grossi, P., DallaGasperina, D., Pagani, F., Marone, P., Vigano, M. and Minoli, L. (2001) Infectious Complications in Patients with the Novacor Left Ventricular Assist System. Transplantation Proceeding, 33, 1969-1971. http://dx.doi.org/10.1161/CIRCULATIONAHA.112.128132

[9] Malani, P.N., Dyke, D.B.S., Pagani, F.D. and Chenoweth, C.E. (2002) Nosocomial Infections in Left Ventricular Assist Device Recipients. Clinical Infectious Diseases, 34, 1295-1300. http://dx.doi.org/10.1086/340052

[10] Sinha, P., Chen, J.M., Flannery, M., Scully, B.E., Oz, M.C. and Edwards, N.M. (2000) Infections during Left Ventricular Assist Device Support Do Not Affect Posttransplant Outcomes. Circulation, 102, III194-III199.

[11] Topkara, V.K., Kondareddy, S., Malik, F., et al. (2010) Infectious Complications in Patients with Left Ventricular Assist Device: Etiology and Outcomes in the Continuous-Flow Era. The Annals of Thoracic Surgery, 90, 1270-1277. http://dx.doi.org/10.1016/j.athoracsur.2010.04.093

[12] Deng, M.C., Loebe, M., El-Banayosy, A., et al. (2001) Mechanical Circulatory Support for Advanced Heart Failure: Effect of Patient Selection on Outcome. Circulation, 103, 231-237. http://dx.doi.org/10.1161/01.CIR.103.2.231

[13] Goldstein, D.J., el-Amir, N.G., Ashton Jr., R.C., et al. (1995) Fungal Infections in Left Ventricular Assist Device Recipients: Incidence, Prophylaxis, and Treatment. ASAIO Journal, 41, 873-875. http://dx.doi.org/10.1097/00002480-199510000-00010

[14] Starling, R.C., Naka, Y., Boyle, A.J., et al. (2011) Results of the Post-US Food and Drug Administration-Approval Study with a Continuous Flow Left Ventricular Assist Device as a Bridge to Heart Transplantation: A Prospective Study Using the INTERMACS (Interagency Registry for Mechanically Assisted Circulatory Support). Journal of the American College of Cardiology, 57, 1890-1898. http://dx.doi.org/10.1016/j.jacc.2010.10.062

[15] Nienaber, J.J., Kusne, S., Riaz, T., et al. (2013) Clinical Manifestations and Management of Left Ventricular Assist Device-Associated Infections. Clinical Infectious Diseases, 57, 1438-1448. http://dx.doi.org/10.1093/cid/cit536

[16] Bomholt, T., Moser, C., Sander, K., et al. (2011) Driveline Infections in Patients Supported with a Heartmate II: Incidence, Aetiology and Outcome. Scandinavian Cardiovascular Journal, 45, 273-278. http://dx.doi.org/10.3109/14017431.2011.577236

[17] Loforte, A., Montalto, A., Ranocchi, F., et al. (2009) HeartmateII Axial-Flow Left Ventricular Assist System: Management, Clinical Review and Personal Experience. Journal of Cardiovascular Medicine (Hagerstown), 10, 765-771. http://dx.doi.org/10.2459/JCM.0b013e32832d495e

[18] John, R., Kamdar, F., Eckman, P., et al. (2011) Lessons Learned from Experience with over 100 Consecutive HeartMateII Left Ventricular Assist Devices. The Annals of Thoracic Surgery, 92, 1593-1599. 
http://dx.doi.org/10.1016/j.athoracsur.2011.06.081

[19] Gordon, R.J., Weinberg, A.D., Pagani, F.D., et al. (2013) Prospective, Multicenter Study of Ventricular Assist Device Infections. Circulation, 127, 691-702. http://dx.doi.org/10.1161/CIRCULATIONAHA.112.128132

[20] Abicht, T., Gordon, R., Meehan, K., Stosor, V., McCarthy, P. and McGee Jr., E. (2013) Complex Heartmate II Infection Treated with Pump Exchange to Heart Ware HVAD. ASAIO Journal, 59, 88-192.

http://dx.doi.org/10.1097/MAT.0b013e318283820d 